\title{
An Adaptive Consensus Reaching Process Dealing with Comparative Linguistic Expressions in Large-scale Group Decision Making
}

\author{
Álvaro Labella ${ }^{a}$ and R. M. Rodríguez ${ }^{b}$ and Luis Martínez ${ }^{c}$ \\ ${ }^{a}$ Department of Computer Science, University of Jaén, alabella@ujaen.es \\ ${ }^{b}$ Department of Computer Science, University of Jaén, rmrodrig@ujaen.es \\ ${ }^{c}$ Department of Computer Science, University of Jaén, martin@ujaen.es
}

\begin{abstract}
Nowadays, society often faces decision problems under uncertainty that can hardly be managed by a single expert or a few of them because of their complexity. Under these conditions, large-scale group decision making (LS-GDM) problems are becoming more and more common. The decisions made on these types of problems might affect directly lots of people in which the consensual decisions are better accepted and consensus reaching processes (CRPs) support reaching such consensus. LS-GDM under uncertainty has been solved by using linguistic information but considering only single linguistic terms to represent experts' opinions. Especially in large-scale, this is an important drawback, since the complexity of the problems causes the apparition of experts' hesitancy, which cannot be modeled by single linguistic terms. Concretely, comparative linguistic expression (CLEs) based on hesitant fuzzy linguistic term sets have provided remarkable results in hesitancy modeling. Therefore, this contribution aims at defining a novel adaptive CRP for LS-GDM in which experts' preferences are modeled by CLEs.
\end{abstract}

Keywords: Large-scale group decision making, consensus reaching process, comparative linguistic expressions.

\section{Introduction}

Human beings deal with decision situations in their daily tasks. Often, these situations become too complex and it is necessary the participation of several experts who facilitate the decision process, under these conditions, we talk about Group Decision Making (GDM). In a GDM problem, experts with different points of view provide their opinions over a set of alternatives in order to select one as solution of the problem [12. Nowadays, the emergence of new technological advances such as social networks 32 or big data 33 and societal needs such as e-democracy [7, 13] or e-marketplace [4, have led to a new GDM concept, socalled Large-scale Group Decision Making (LS-GDM), which has attracted the researchers' attention in decision making area [15, 18, 26]. Whereas classical GDM problems only require the participation of a few experts in the decision process, LS-GDM problems make necessary the engagement of a greater number of them. Despite the fact that there is no a definitive consensus in the literature 8, a GDM is considered large-scale when the number of experts is greater than 20, although the number might be much greater. LS-GDM problems present unique characteristics resulting in new challenges to face, for instance: (i) scalability, (ii) minority opinions, (iii) non-cooperative behaviors, (iv) opinion polarization etc.

LS-GDM problems are usually related to decisions that affect society. In this situation, consensual solutions are more appreciated. For this reason, Consensus Reaching Processes (CRPs) are included as an additional phase in the resolution of LS-GDM problems [31. In a CRP process, experts discuss among them and modify their initial opinions in order to achieve an agreed solution. Most of the proposed CRPs assume just a few number of experts [10, although the larger number of experts implies more conflicts and disagreements and thus, a greater necessity of applying a CRP. On the other hand, another aspect that has direct influence on the GDM problems complexity is their inherent uncertainty related to vagueness and imprecision and which provokes vague opinions of the elicited information, usually modeled by linguistic descriptors 21. Particularly, uncertainty in LS-GDM is quite common and, consequently, experts' hesitancy, due to the complexity of the problems. However, most of linguistic based CRPs proposed deal with linguistic information represented just for single linguistic terms 
in which it is not possible to reflect experts' hesitancy [22, 34]. The latter drawback can be managed by using more complex and flexible linguistic expressions such as Comparative Linguistic Expressions (CLEs), based on Hesitant Fuzzy Linguistic Term Sets (HFLTSs) 28, which allow to model experts' hesitancy by means of linguistic expressions closer to human beings' cognitive process. Despite there are several proposals about CRP which deal with HFLTSs [9, 23, any of them consider their fuzzy representation and even less are focused on LS-GDM problems.

Bearing in mind the foregoing, this paper aims at defining a novel adaptive CRP for LS-GDM that models the experts' hesitancy by means of CLEs based on HFLTS by using their fuzzy representation.

This paper is structured as follows: Section 2 reviews briefly concepts related to GDM, CRP, HFLTs and CLEs in order to facilitate the understanding of the proposal. Section 3 presents a novel adaptive CRP for LS-GDM dealing with linguistic information represented by CLEs. Section 4 introduces a real LS-GDM problem in order to show the usefulness and validity of the proposed CRP. Finally, in Section 5 some conclusions are drawn.

\section{Preliminaries}

This section reviews different concepts related to GDM, CRPs, HFLTS and CLEs.

\subsection{Group Decision Making}

GDM is a process formed by a set of experts with their own attitudes who try to reach a common solution for a decision problem by selecting an alternative or solution among of set of them 20. Formally, a GDM problem is characterized by a set of experts $E=\left\{e_{1}, \ldots e_{m}\right\}$ who express their opinions over a set of alternatives $X=\left\{x_{1}, \ldots x_{n}\right\}$ [1]. Each expert $e_{i}$ provides his/her preferences over pair of alternatives $\left(x_{l}, x_{j}\right)$ by using preference relations, $P^{i}=\left(p_{l j}^{i}\right)_{n \times n}$.

Although GDM concept has been widely used in decision theory [2, 6, at the present time society demands decision processes in which the participation of crowds is required. GDM problems and LS-GDM have several similarities but also significant differences. The number of experts who participate in the latter are much bigger than the former and, in addition, much larger than the number of alternatives $(m>>n)$. On the other hand, both types of problems can be solved in a similar way by a resolution process composed by two main phases, aggregation and exploitation [30. However, such resolution process does not guarantee to reach solutions accepted by all the experts and several of them may have the feeling that their opinions have not been sufficiently taken into account 31. To solve the latter issue, CRPs have been included as an additional phase in the resolution scheme.

\subsection{Consensus Reaching Process}

A CRP is an iterative process in which experts who participate in a decision process discuss among them and change their initial opinions by trying to make their opinions closer to each other in order to achieve a consensus 31. Consensus concept has been widely discussed in the literature [5], some researchers consider consensus as unanimity, hardly attainable in realworld problems. Other views are less strict, being soft consensus one of the most extended and accepted [11]. Kacprzyk exposed that consensus is reached when "most of the important individuals agree (as to their testimonies concerning) almost all of relevant opinions".

CRPs aim to achieve a high level of agreement after several discussion rounds. The process is guided by a person, so-called moderator, whose main task is to identify sticking points in the experts' group and solve them making their opinions are closer each other. Fig. 1 shows a general scheme for CRP proposed in 24$]$ whose phases are detailed below.

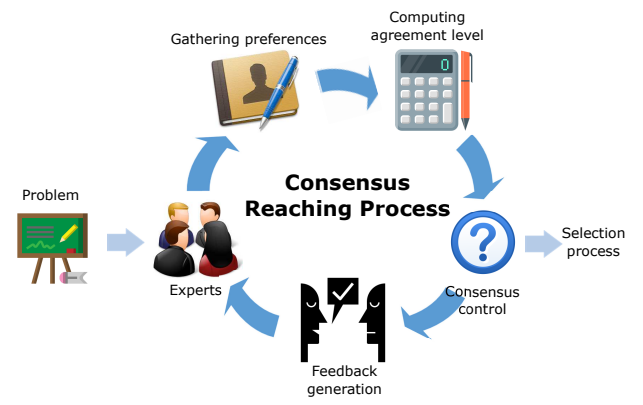

Figure 1: CRP scheme.

1. Gathering preferences: experts study the decision problem and express their preferences over the different alternatives by preference relations.

2. Computing agreement level: the level of agreement in the experts' group is computed.

3. Consensus control: the current level of agreement is compared to a predefined consensus threshold, which represents the minimum level of agreement to be reached. If consensus threshold is achieved, a selection process of the best alternative starts, otherwise it is necessary another discussion round.

4. Feedback generation: moderator identifies the furthest experts' opinions in the group and advises to them to modify their opinions, increasing the level of agreement. 


\subsection{Hesitant Fuzzy Linguistic Term Sets and Comparative Linguistic Expressions}

Uncertainty frequently appears in GDM problems due to the lack of information and vagueness. Under these conditions, linguistic information has obtained successful results to model such uncertainty. Classically, experts have used single linguistic terms to provide their preferences, but this might be an important drawback, since the lack of information and uncertainty implies a greater experts' hesitancy that cannot be represented in that way. HFLTSs 29] were defined in order to facilitate the experts' preferences elicitation when they doubt among several linguistic terms (see Fig. 2).

Definition 1 [29] Let $S=\left\{s_{0}, \ldots, s_{g}\right\}$ be a ordered linguistic term set, a HFLTS, $H_{S}$, is an ordered finite subset of consecutive linguistic terms of $S$.

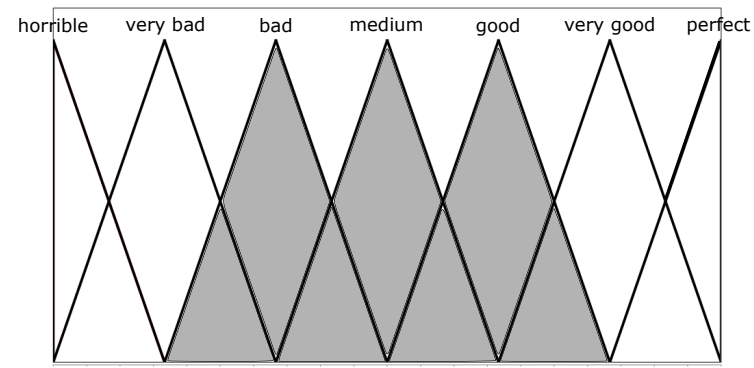

Figure 2: HFLTS.

HFLTs make easier the experts' preferences elicitation and allow to model experts' hesitancy but they are quite far from the way in which human being's express their knowledge or preferences. For this reason, several proposals have introduced complex linguistic expressions closer to the human beings' cognitive process 25. However, CLEs 27, 29] stand out because of their interpretability. CLEs are based on HFLTSs and are generated by means of a context-free grammar 25. Some examples of CLEs are: between good and very good, at least bad or at most medium.

CLEs represent linguistic information in a more comprehensive way, but it is fundamental to carry out computations with these expressions in order to solve GDM problems. For this reason, a transformation function, $E_{G_{H}}$, which transforms CLEs into HFLTSs was also proposed in 29. The possible transformations of CLEs into HFLTs are:

$$
\begin{aligned}
& E_{G_{H}}\left(s_{i}\right)=\left\{s_{i} \mid s_{i} \in S\right\} \\
& E_{G_{H}}\left(\text { at most } s_{i}\right)=\left\{s_{j} \mid s_{j} \leq s_{i} \text { and } s_{j} \in S\right\} \\
& E_{G_{H}}\left(\text { at least } s_{i}\right)=\left\{s_{j} \mid s_{j} \geq s_{i} \text { and } s_{j} \in S\right\} \\
& E_{G_{H}}\left(\text { between } s_{i} \text { and } s_{j}\right)=\left\{s_{k} \mid s_{i} \leq s_{k} \leq s_{j}\right. \\
& \text { and } \left.s_{k} \in S\right\}
\end{aligned}
$$

Once CLEs are transformed into HFLTSs, several computational models have proposed to accomplish the linguistic computations. This contribution makes use of the fuzzy envelope concept, which represents the semantics of the CLEs by means of trapezoidal membership functions from the linguistic terms belonging to the respective HFLTS (see [19] for further details).

Definition 2 [19] The fuzzy envelope, env ${ }_{F}\left(H_{S}\right)$, is defined as a trapezoidal fuzzy membership function, $T(a, b, c, d)$, such that

$$
e n v_{F}\left(H_{S}\right)=T(a, b, c, d)
$$

\section{An Adaptive Consensus Model for LS-GDM based on CLEs}

This section introduces a novel adaptive consensus model able to face the challenges in LS-GDM such as scalability and time cost. Furthermore, experts' preferences are elicited by CLEs, which are subsequently transformed into HFLTSs, which in turn, are modeled by their fuzzy envelopes, keeping the fuzzy representation and used to achieve the required level of agreement. The proposal modifies and adds new phases to the classical CRP model (see Fig. 1) that are shown in Fig. 3 and further explained in the coming subsections.

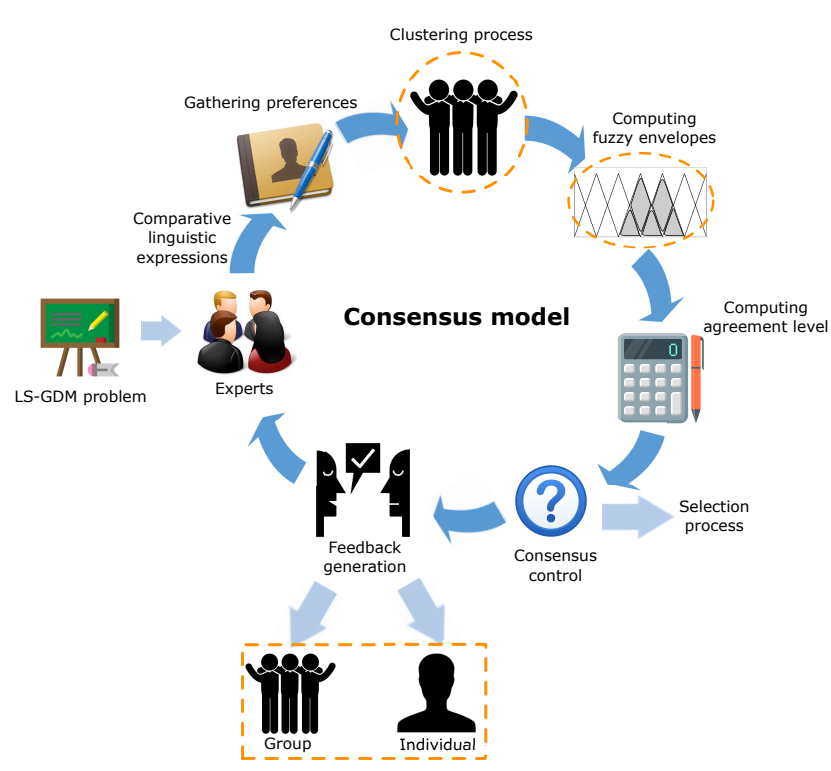

Figure 3: Proposed CRP scheme.

\subsection{Gathering preferences}

For the proposed consensus model, experts express their preferences by CLEs. Concretely, each expert $e_{i}$ expresses his/her preferences by using a hesitant linguistic preference relation (HLPR) 35], $P^{i}, X \times X \rightarrow$ 
$S_{l l}$, where $S_{l l}$ is the set of CLEs generated by using the linguistic terms belonging to the linguistic term set $S$. Considering $S$ the linguistic terms set represented in Fig. 2, an example of HLPR may be:

$$
P^{i}=\left(\begin{array}{ccc}
- & \text { medium } & \text { good } \\
\text { medium } & - & \text { at most bad } \\
\text { bad } & \text { bt medium and good } & -
\end{array}\right)
$$

Remark 1 bt stands for between.

\subsection{Clustering process}

The large number of experts in LS-GDM problems implies scalability problems. To tackle the scalability problem in LS-GDM, we consider applying a clustering process in which experts will be divided into subgroups/clusters composed by those experts with similar opinions, by reducing the number of preferences to manage. The clustering algorithm applied in this proposal is the fuzzy c-means [1, widely used in the literature. For sake of space, such algorithm is not introduced here, but in a brief summary, this algorithm computes iteratively cluster centers or centroids, which represent all data objects belonging to a same cluster, and assigns a membership degree to each data object for each cluster according to the distance between such data object and the corresponding centroid. A relevant step in fuzzy c-means is the initialization of the clusters and the representation of their respective centroids. In this contribution, the number of clusters, $N$, will be equal to the number of alternatives, with the aim of finding the clusters of experts supporting each different alternative. Consequently, each centroid, $C^{k}$, will be initialized with a HLPR that represents the total preference of the corresponding alternative over all the others.

For each iteration, $t$, the centroids $C^{k}$ are computed together with the membership degree of each expert' preference $P^{i}$ to each centroid $C^{k}$ such that:

$$
\mu_{C^{k, t}}\left(P^{i}\right)=\frac{\left(1 / d_{H}\left(P^{i}, c^{k, t}\right)\right)^{2 /(b-1)}}{\sum_{u=1}^{n}\left(1 / d_{H}\left(P^{i}, c^{u, t}\right)\right)^{2 /(b-1)}}
$$

where $d_{H}(\cdot)$ is a distance measure etween two HLPRs [35], $t$ is the current iteration, and $b$ indicates the fuzziness degree of the clusters. The larger $b$, the fuzzier the clusters 1 .

\subsection{Computing fuzzy envelopes}

The assessments, $p_{l j}^{i}$, provided by each expert $e_{i}$ are transformed into HFLTSs 29] and subsequently modeled by their respective fuzzy envelope (1), $p_{l j}^{i}=$ $T(a, b, c, d)$. In the same way, the assessments of the centroids of each subgroup $C^{k}$ are also transformed into fuzzy envelopes, $c_{l j}^{k}=T(a, b, c, d)$.

\subsection{Computing consensus degree}

The consensus degree $c r \in[0,1]$, which measures the level of agreement in the group of experts is the basis for the adaptability of the CRP and it is computed as:

1. Compute similarity matrices: for each pair of experts $\left(e_{i}, e_{t}\right), i<t$, a similarity matrix $S M^{i t}=$ $\left(s m_{l j}^{i t}\right)_{n \times n}$ is obtained. Each similarity value $s m_{l j}^{i t} \in[0,1]$ represents the agreement level between the experts $e_{i}$ and $e_{t}$ about the pair of alternatives $\left(x_{l}, x_{j}\right)$ :

$$
s m_{l j}^{i t}=1-\operatorname{dist}_{T}\left(p_{l j}^{i}, p_{l j}^{t}\right)
$$

where $\operatorname{dist}_{T}(\cdot)$ is a distance measure between two trapezoidal fuzzy numbers [17].

2. Compute consensus matrix: the similarity values are aggregated by means of an aggregation operator, $\rho$, by obtaining a consensus matrix $C M=$ $\left(c m_{l j}\right)_{n \times n}$.

$$
c m_{l j}=\rho\left(S I M_{l j}\right)
$$

where $S I M_{l j}$ represents the set of all pairs of experts' similarities about the pair of alternatives $\left(x_{l}, x_{j}\right)$ with $\left|S I M_{l j}\right|=\left(\begin{array}{c}m \\ 2\end{array}\right)$, and $c m_{l j}$ the consensus degree achieved by the group of experts about the pair of alternatives $\left(x_{l}, x_{j}\right)$.

3. Compute alternatives consensus degree: from $C M$, a consensus degree $c a^{l}$ is computed for each alternative $x_{l}$.

$$
c a^{l}=\frac{\sum_{j=1, j \neq l}^{n} c m_{l j}}{n-1}
$$

4. Compute overall consensus degree: overall consensus degree, $c r$, is computed as follows:

$$
c r=\frac{\sum_{l=1}^{n} c a^{l}}{n}
$$

\subsection{Consensus control}

The overall consensus degree, $c r$, is compared with a predefined consensus threshold, $\alpha \in[0,1]$, that represents the required consensus. If $c r \geq \alpha$ a selection process of the best alternative starts, otherwise, the CRP requires another discussion round. The number of rounds is usually limited by another predefined parameter, Maxrounds $\in \mathbb{N}$.

\subsection{Feedback generation}

In case that $c r<\alpha$, it is necessary to increase the level of agreement between experts by means of a feedback process. In order to reduce the time cost, our proposal adapts the feedback process according to the consensus 
level achieved and a predefined threshold $\sigma$. If $\mathrm{cr}<\sigma$, a feedback process for groups is applied, otherwise the feedback process is applied for single experts. The feedback process consist of:

1. Compute the collective opinion: a collective matrix that represents the overall opinion of all the experts involved in the LS-GDM problem is computed for each pair of alternatives by aggregating the centroids of each subgroup, $C=\left\{C^{1}, \ldots C^{n}\right\}$, by means of a fuzzy aggregation operator, $\lambda$.

$$
p_{l j}^{c}=\lambda\left(c_{l j}^{1}, \ldots c_{l j}^{n}\right)
$$

2. Compute proximity matrices: a proximity matrix $P P^{k}$ between the subgroup $C^{k}$ and the collective opinion $P^{c}$ is computed as:

$$
p p_{l j}^{k}=1-d_{T}\left(c_{l j}^{k}, p_{l j}^{c}\right)
$$

Proximity values, $p p^{k}$, are used to identify the subgroups that are furthest from the collective opinion.

Depending on the consensus level reached $c r$, the feedback process will be aimed at all experts of the furthest subgroups or just for several experts.

- Group feedback process: in this case, $c r<\sigma$, then consensus is "low" and quite more changes are necessary. Consequently, the experts belonging to the furthest subgroups are recommended to change their preferences over the pair of alternatives identified in disagreement. To obtain the furthest subgroups and the pair of alternative to change, the proximity value of each subgroup is compared with the average of the proximity values, $\overline{p p}_{l j}$ such that:

$$
\overline{p p}_{l j}=\frac{1}{n} \sum_{u=1}^{n} p p_{l j}^{u}
$$

If $c a^{l}<\alpha$ and $p p_{l j}^{k}<\overline{p p}_{l j}$ then, the preferences on the pair of alternatives $\left(x_{l}, x_{j}\right)$ should be changed for the experts' preferences belonging to the subgroup $G^{k}$.

- Individual feedback process: in this case, $\mathrm{cr}>$ $\sigma$, then consensus is "high" but not enough and not many changes are necessary. Consequently, the furthest experts from the collective opinion should change their preferences. The subgroup and the pair of alternative to change are identified in the same way that in the group feedback process but, in this occasion, an expert $e^{i} \in G^{k}$ who should change his/her preferences will be the one who $\left(1-d_{T}\left(p_{l j}^{c}, p_{l j}^{i}\right) \leq \overline{p p}_{l j}\right)$.
Finally, once the modifications for the experts are identified, the last step is to identify in which direction experts should modify their assessments. Several direction rules are applied to suggest the direction of the changes in order to increase the level of agreement in the group. To do so, an acceptability threshold $\varepsilon \geq 0$, a positive value close to zero, defines a margin of acceptability when $p_{l j}^{i}$ and $p_{l j}^{c}$ are close to each other.

- RULE 1: If $\delta\left(p_{l j}^{i}\right)-\delta\left(p_{l j}^{c}\right)<-\varepsilon$ then $e_{i} \in$ $G^{k}$, should increase his/her assessments $p_{l j}^{i}$ on $\left(x_{l}, x_{j}\right)$.

- RULE 2: If $\delta\left(p_{l j}^{i}\right)-\delta\left(p_{l j}^{c}\right)>\varepsilon$ then $e_{i} \in$ $G^{k}$ should decrease his/her assessments $p_{l j}^{i}$ on $\left(x_{l}, x_{j}\right)$.

- RULE 3: If $-\varepsilon \leq \delta\left(p_{l j}^{i}\right)-\delta\left(p_{l j}^{c}\right) \leq \varepsilon$ then $e_{i} \in$ $G^{k}$ should not modify his/her assessments $p_{l j}^{i}$ on $\left(x_{l}, x_{j}\right)$.

where $\delta(\cdot)$ denotes the defuzzified value of a trapezoidal fuzzy membership function $T(a, b, c, d)$ such that:

$$
\delta(T(a, b, c, d))=\frac{(a+2 b+2 c+d)}{6}
$$

Previous rules identify the change direction but not how the change should be applied. According to the direction of the change received and taking into account that experts accept the suggestion provided by the consensus model, the change to apply is defined as follows:

- Expert $e_{i}$ should increase his/her assessment $p_{l j}^{i}$.

- If $p_{l j}^{i}=s_{p}$, where $s_{p}$ is a single linguistic term, then the recommendation for the expert is to change his/her assessment so that $p_{l j}^{i}=s_{p+\theta}, \theta \in[1, g-1], p+\theta \leq g$. In case that $s_{p}=s_{g}$ no change will be applied.

- If $p_{l j}^{i}=$ at least $s_{p}$ or at most $s_{p}$, where $s_{p}$ is a linguistic term, then the recommendation for the expert is to change his/her assessment so that $p_{l j}^{i}=$ at least $s_{p+\theta}$ or at most $s_{p+\theta}$ respectively, $\theta \in[1, g-1], p+\theta \leq g$. In case that $s_{p}=s_{g}$ no change will be applied.

- If $p_{l j}^{i}=$ between $s_{p}$ and $s_{q}$, where $s_{p}, s_{q}$ are linguistic terms $p>q$, then the recommendation for the expert is to change his/her assessment so that $p_{l j}^{i}=$ between $s_{p+\theta}$ and $s_{q}$, $\theta \in[1, g-1], p+\theta \leq g$ and $p+\theta \leq q$. In case that $s_{p+\theta}=s_{q}$, the new assessment is $p_{l j}^{i}=s_{q}$

- Expert $e_{i}$ should decrease his/her assessment $p_{l j}^{i}$. 
- If $p_{l j}^{i}=s_{p}$, where $s_{p}$ is a single linguistic term, then the recommendation for the expert is to change his/her assessment so that $p_{l j}^{i}=s_{p-\theta}, \theta \in[1, g-1], p-\theta \geq 0$. In case that $s_{p}=s_{0}$ no change will be applied.

- If $p_{l j}^{i}=$ at least $s_{p}$ or at most $s_{p}$, where $s_{p}$ is a linguistic term, then the recommendation for the expert is to change his/her assessment so that $p_{l j}^{i}=$ at least $s_{p-\theta}$ or at most $s_{p-\theta}$ respectively, $\theta \in[1, g-1], p-\theta \geq 0$. In case that $s_{p}=s_{0}$ no change will be applied.

- If $p_{l j}^{i}=$ between $s_{p}$ and $s_{q}$, where $s_{p}, s_{q}$ are linguistic terms $p>q$, then the recommendation for the expert is to change his/her assessment so that $p_{l j}^{i}=$ between $s_{p}$ and $s_{q-\theta}$, $\theta \in[1, g-1], q-\theta \geq 0$ and $q-\theta \geq p$. In case that $s_{q}=s_{0}$, no change will be applied.

Remark 2 The parameter $\theta \in \mathbb{N}^{[1, g-1]}$ expresses the change degree to apply, which can be adjusted depending on the desired degree.

\section{Case Study}

This section introduces a real-world LS-GDM problem that is applied to the proposed adaptive CRP, demonstrating their advantages and qualities.

Let us suppose a panel of experts consisting of 50 members, $E=\left\{e_{1}, \ldots e_{50}\right\}$ who has the difficult task to decide which city will be in charge of organizing 2024 Winter Olympic Games. After a exhaustive preselection, just three cities are candidates to win, $X=\left\{x_{1}:\right.$ Moscow, $x_{2}:$ Oslo, $x_{3}:$ Vancouver $\}$. To solve this problem, the following parameters has been considered:

- Consensus threshold: $\alpha=0.85$.

- Level of consensus for advice generation: $\sigma=0.7$.

- Acceptability threshold: $\varepsilon=0.05$.

- Maximum number of rounds: Maxrounds $=15$.

Following the steps introduced in Section 3

1. Gathering preferences: each expert expresses his/her assessments over the three cities by HFLPRs.

2. Clustering process: The fuzzy c-means algorithm is applied to obtain the clustering containing the subgroups of experts with similar opinions. Table 1 shows the experts' subgroups in the first round, $\left\{G^{1}, G^{2}, G^{3}\right\}$, one for each alternative.

\begin{tabular}{|c|c|}
\hline$G^{k}$ & EXPERTS \\
\hline$G_{1}$ & $\left\{e_{1}, e_{3}, e_{5}, e_{6}, e_{7}, e_{13}, e_{18}, e_{19}, e_{20}\right.$, \\
& $\left.e_{23}, e_{33}, e_{35}, e_{38}, e_{42}, e_{45}, e_{46}, e_{47}, e_{50}\right\}$ \\
\hline$G_{2}$ & $\left\{e_{2}, e_{9}, e_{10}, e_{12}, e_{16}, e_{17}, e_{25}\right.$, \\
& $\left.e_{27}, e_{32}, e_{34}, e_{37}, e_{43}, e_{44}, e_{49}\right\}$ \\
\hline$G_{3}$ & $\left\{e_{4}, e_{8}, e_{11}, e_{14}, e_{15}, e_{21}, e_{22}, e_{24}, e_{26}\right.$, \\
$\left.e_{28}, e_{29}, e_{30}, e_{31}, e_{36}, e_{39}, e_{40}, e_{41}, e_{48}\right\}$ \\
\hline
\end{tabular}

Table 1: Experts' subgroups in the first round.

3. Computing fuzzy envelopes: Each assessment $p_{l j}^{i}$ provided by expert $e_{i}$ is transformed into a HFLTS and subsequently to a trapezoidal fuzzy membership function by computing its respective fuzzy envelope. In the same way, for the centroids of the subgroups.

4. Computing consensus degree: the consensus degree, $c r$, achieved for each round is shown in Table 2 ,

\begin{tabular}{|c|c|c|}
\hline ROUND & $c r$ & Level \\
\hline Initial & 0.6 & Low \\
1 & 0.64 & Low \\
2 & 0.72 & High \\
3 & 0.74 & High \\
4 & 0.76 & High \\
5 & 0.85 & High \\
\hline
\end{tabular}

Table 2: Consensus degree achieved in each round.

5. Consensus control: according to the results shown in Table 2, the consensus model needs 5 rounds to reach the desired consensus level $\alpha$. Therefore, the selection process of the best alternative starts in round 5 .

6. Feedback generation: according to the results shown in Table 2, a group feedback process is carried out in round 1 , since $c r<\sigma$. In this moment, the furthest subgroups are identified and recommended to change their preferences in order to increase the level of agreement. From here, an individual feedback process is applied in the following rounds, since $\mathrm{cr}>\sigma$. In this situation, the furthest subgroups are identified and, within them, the specific experts who should change their opinions. Fig 4 shows the evolution of the CRP obtained from the framework AFRYCA 3.0 16] by means of a Muti-Dimensional Scaling (MDS) [14] visualization of the experts' preferences.

Analyzing the results, it has been demonstrated that the proposed adaptive consensus model performs ef- 


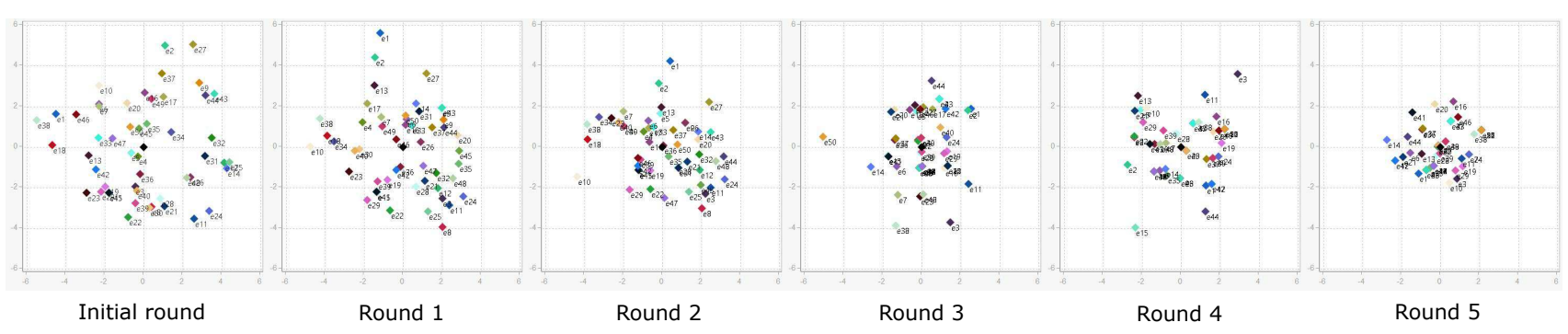

Figure 4: MDS visualization of the CRP.

fectively the CRP in LS-GDM. The adaptive feedback process allows to obtain a solution in a few number of discussions rounds, reducing time cost. Furthermore, the clustering process reduces the scalability problems related to LS-GDM problems. Therefore, two of the most relevant challenges in LS-GDM are managed properly. Last but not least, consensus model deals with linguistic information represented by CLEs managing their fuzzy representation, in this way, we provide an effective consensus model that allows experts express their opinions in a understandable and intuitive way.

\section{Conclusions}

Decision situations evolve at the same time as society's needs evolve. This evolution has led to the LS-GDM problems, in which a large number of experts is necessary to make the decision. The inherent complexity of these problems results in a frequent hesitancy from experts, which makes it necessary the use of complex linguistic expressions, such as CLEs, which allow to model such hesitancy and facilitate the experts' preferences elicitation. Furthermore, society demands consensual solution to guarantee that the decisions made are reasonable and fair. Notwithstanding the foregoing, most of current proposals in literature are focused on CRPs for group decision situations with a few number of experts.

A novel adaptive CRP for LG-GDM in which experts' preferences are modeled by CLEs has been introduced. The CRP introduces a formalized feedback generation which considers the fuzzy representation of the CLEs and applies the changes directly on the CLEs, keeping their interpretability. The proposal is accompanied by a case study to show its validity and usefulness in the resolution of LS-GDM problems.

\section{Acknowledgement}

This work is partially supported by the Spanish National Research Project TIN2015-66524-P and the Postdoctoral fellow Ramón y Cajal (RYC-201721978), Spanish Ministry of Economy and Competitiveness.

\section{References}

[1] J. Bezdek, Pattern Recognition with Fuzzy Objective Function Algorithms, Springer, 1981.

[2] J. Bezdek, B. Spillman, R. Spillman, A fuzzy relation space for group decision theory, Fuzzy sets and Systems 1 (4) (1978) 255-268.

[3] C. Butler, A. Rothstein, On Conflict and Consensus: A Handbook on Formal Consensus Decision Making, Takoma Park, 2006.

[4] G. Büyüközkan, Multi-criteria decision making for e-marketplace selection, Internet Research 14 (2) (2004) 139-154.

[5] F. Cabrerizo, J. Moreno, I. Perez, E. HerreraViedma, Analyzing consensus approaches in fuzzy group decision making: advantages and drawbacks, Soft Computing 15 (5) (2010) 451-463.

[6] N. Capuano, F. Chiclana, H. Fujita, E. HerreraViedma, V. Loia, Fuzzy group decision making with incomplete information guided by social influence, IEEE Transactions on Fuzzy Systems 26 (3) (2018) 1704-1718.

[7] A. Chadwick, Web 2.0: New challenges for the study of e-democracy in an era of informational exuberance, I/S: A Journal of Law and Policy for the Information Society 5 (1) (2009) 9-41.

[8] X. Chen, R. Liu, Improved clustering algorithm and its application in complex huge group decision-making, Systems Engineering and Electronics 28 (11) (2006) 1695-1699.

[9] Y. Dong, X. Chen, F. Herrera, Minimizing adjusted simple terms in the consensus reaching process with hesitant linguistic assessments in group decision making, Information Sciences 297 (2015) 95-117.

[10] E. Herrera-Viedma, F. Cabrerizo, J. Kacprzyk, W. Pedrycz, A review of soft consensus models in a fuzzy environment, Information Fusion 17 (2014) $4-13$. 
[11] J. Kacprzyk, Group decision making with a fuzzy linguistic majority, Fuzzy Sets and Systems 18 (2) (1986) 105-118.

[12] J. Kacprzyk, M. Fedrizzi, H. Nurmi, Group decision making and consensus under fuzzy preferences and fuzzy majority, Fuzzy Sets and Systems 49 (1) (1) (1992) 21-31.

[13] J. Kim, A model and case for supporting participatory public decision making in e-democracy, Group Decision and Negotiation 17 (3) (2008) 179-192.

[14] J. Kruskal, M. Wish, Multidimensional scaling, Springer, 1978.

[15] Á. Labella, Y. Liu, R. M. Rodríguez, L. Martínez, Analyzing the performance of classical consensus models in large scale group decision making: A comparative study, Applied Soft Computing 67 (2018) 677-690.

[16] A. Labella, L. Martínez, AFRYCA 3.0: An improved framework for consensus analysis in group decision making, in: International Conference on Intelligent Decision Technologies, Springer, 2018, pp. $76-86$.

[17] H. Lee, Optimal consensus of fuzzy opinions under group decision making environment, Fuzzy sets and systems 132 (1) (2002) 303-315.

[18] B. Liu, Y. Shen, Y. Chen, X. Chen, Y. Wang, A two-layer weight determination method for complex multi-attribute large-group decision-making experts in a linguistic environment, Information Fusion 23 (2015) $156-165$.

[19] H. Liu, R. M. Rodríguez, A fuzzy envelope for hesitant fuzzy linguistic term set and its application to multicriteria decision making, Information Sciences 258 (2014) 220-238.

[20] J. Lu, D. Ruan, Multi-objective group decision making: methods, software and applications with fuzzy set techniques, Vol. 6, Imperial College Press, 2007.

[21] L. Martínez, D. Ruan, F. Herrera, E. HerreraViedma, P. Wang, Linguistic decision making: Tools and applications, Information Sciences 179 (14, Spec. Iss.) (2009) $2297-2298$.

[22] F. Mata, L. Martínez, E. Herrera-Viedma, An adaptive consensus support model for group decision-making problems in a multigranular fuzzy linguistic context, IEEE Transactions on Fuzzy Systems 17 (2) (2009) 279-290.
[23] J. Montserrat-Adell, N. Agell, M. Sanchez, F. Javier Ruiz, Consensus, dissension and precision in group decision making by means of an algebraic extension of hesitant fuzzy linguistic term sets', Information Fusion 42 (2018) 1-11.

[24] I. Palomares, F. Estrella, L. Martínez, F. Herrera, Consensus under a fuzzy context: Taxonomy, analysis framework AFRYCA and experimental case of study, Information Fusion 20 (November 2014) (2014) 252-271.

[25] R. Rodríguez, A. Labella, L. Martínez, An overview on fuzzy modelling of complex linguistic preferences in decision making, International Journal of Computational Intelligence Systems 9 (2016) 81-94

[26] R. M. Rodríguez, A. Labella, G. D. Tré, L. Martínez, A large scale consensus reaching process managing group hesitation, KnowledgeBased Systems.

[27] R. M. Rodríguez, L. Martínez, An analysis of symbolic linguistic computing models in decision making, International Journal of General Systems 42 (1) (2013) 121-136.

[28] R. M. Rodríguez, L. Martínez, F. Herrera, Hesitant fuzzy linguistic term sets for decision making, IEEE Transactions on Fuzzy Systems 20 (1) (2012) 1109-119.

[29] R. M. Rodríguez, L. Martínez, F. Herrera, A group decision making model dealing with comparative linguistic expressions based on hesitant fuzzy linguistic term sets, Information Sciences 241 (1) (2013) 28-42.

[30] M. Roubens, Fuzzy sets and decision analysis, Fuzzy Sets and Systems 90 (2) (1997) 199-206.

[31] S. Saint, J. R. Lawson, Rules for Reaching Consensus. A Modern Approach to Decision Making, Jossey-Bass, 1994.

[32] C. Sueur, J.-L. Deneubourg, O. Petit, From social network (centralized vs. decentralized) to collective decision-making (unshared vs. shared consensus), PLoS one 7 (2) (2012) e32566.

[33] S. J. Walker, Big data: A revolution that will transform how we live, work, and think (2014).

[34] Z. Wu, J. Xu, Consensus reaching models of linguistic preference relations based on distance functions, Soft Computing 16 (4) (2012) 577-589.

[35] B. Zhu, Z. Xu, Consistency measures for hesitant fuzzy linguistic preference relations, IEEE Transactions on Fuzzy Systems 22 (1) (2014) 35-45. 